\title{
$5-1-2011$
}

\section{The variable presentations of anaplastic spindle cell squamous carcinoma associated with tall cell variant of papillary thyroid carcinoma.}

\author{
Pallavi P Gopal \\ Department of Pathology and Laboratory Medicine, University of Pennsy/vania \\ Kathleen T Montone \\ Department of Pathology and Laboratory Medicine, University of Pennsylvania \\ Zubair Baloch \\ Department of Pathology and Laboratory Medicine, University of Pennsylvania \\ Madalina Tuluc \\ Department and additional works at: https://jdc.jefferson.edu/pacbfp \\ Department of Pathology, Thomas Jefferson University \\ Part of the Medical Cell Biology Commons, and the Pathology Commons

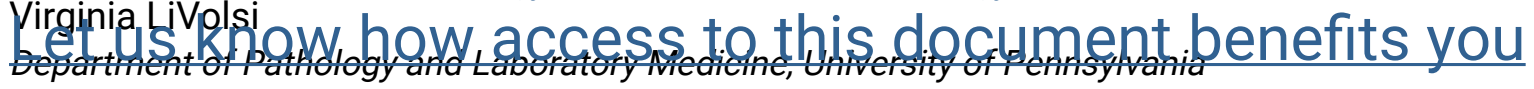

\section{Recommended Citation}

Gopal, Pallavi P; Montone, Kathleen T; Baloch, Zubair; Tuluc, Madalina; and LiVolsi, Virginia, "The variable presentations of anaplastic spindle cell squamous carcinoma associated with tall cell variant of papillary thyroid carcinoma." (2011). Department of Pathology, Anatomy, and Cell Biology Faculty Papers. Paper 78.

https://jdc.jefferson.edu/pacbfp/78

This Article is brought to you for free and open access by the Jefferson Digital Commons. The Jefferson Digital Commons is a service of Thomas Jefferson University's Center for Teaching and Learning (CTL). The Commons is a showcase for Jefferson books and journals, peer-reviewed scholarly publications, unique historical collections from the University archives, and teaching tools. The Jefferson Digital Commons allows researchers and interested readers anywhere in the world to learn about and keep up to date with Jefferson scholarship. This article has been accepted for inclusion in Department of Pathology, Anatomy, and Cell Biology Faculty Papers by an authorized administrator of the Jefferson Digital Commons. For more information, please contact: JeffersonDigitalCommons@jefferson.edu. 


\title{
The Variable Presentations of Anaplastic Spindle Cell Squamous Carcinoma Associated with Tall Cell Variant of Papillary Thyroid Carcinoma
}

\author{
Pallavi P. Gopal, ${ }^{1}$ Kathleen T. Montone, ${ }^{1}$ Zubair Baloch, ${ }^{1}$ Madalina Tuluc, ${ }^{2}$ and Virginia LiVolsi ${ }^{1}$
}

Background: In 1976, Hawk and Hazard described the tall cell variant (TCV) of papillary thyroid carcinoma (PTC). While the lesions they described had cytologic features of papillary carcinoma, they showed more aggressive behavior with a greater propensity for extrathyroid extension and lymphovascular invasion than classic PTC. In 1991, Bronner and LiVolsi described a series of patients with TCV that progressed to spindle cell squamous carcinoma (SCSC), a unique form of anaplastic thyroid carcinoma. This study describes the variable clinical and pathologic presentations in 31 patients with anaplastic SCSC arising in association with TCV.

Methods: The surgical pathology archives as well as the personal consultation files of one of the authors (V.A.L.) were reviewed to retrieve cases of SCSC arising in association with TCV. The available clinical as well as pathologic information on all patients was reviewed.

Results: A total of 31 patients with SCSC arising in association with TCV were retrieved from our files. The average age at primary presentation was 67 (range 32-92) with a female-to-male ratio of 2:1. Three clinical scenarios for SCSC associated with TCV were identified. These were type I, consisting of TCV with SCSC at the time of presentation (18 patients); type II, consisting of SCSC arising as a recurrence in patients with a known history of TCV (5 patients); and type III, consisting of SCSC presenting as a primary laryngeal squamous cell carcinoma in a patient with or without a known history of TCV (8 patients). The type III cases were of most concern since they often were confused with primary laryngeal squamous cell carcinoma and most often were diagnosed after laryngectomy.

Conclusions: SCSC of the thyroid is almost exclusively associated with TCV and can have variable clinical presentations. SCSC is most commonly seen associated with a primary diagnosis of TCV. SCSC may be seen, however, in patients with recurrent PTC and most importantly may present in a fashion similar to primary laryngeal SCSC. Therefore, caution should be exercised in evaluating laryngeal squamous lesions in patients with known history of TCV and without known risks factors for head and neck carcinogenesis.

\section{Introduction}

T

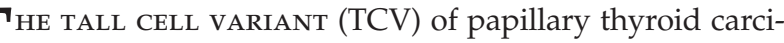
noma (PTC), first described by Hawk and Hazard in 1976, has been defined as a tumor composed of $>50 \%$ cells whose heights are at least two or three times their widths and have nuclear features of PTC $(1,2)$. TCV tends to occur in older patients, forms larger and bulkier tumor masses, is more likely to show extrathyroidal extension, and shows more aggressive behavior, suggesting that TCV has a pathobiology distinct from classic PTC $(1,3)$.

In addition, Bronner and LiVolsi and others have noted that some cases of TCV progress to spindle cell squamous carci- noma (SCSC), an unusual form of anaplastic carcinoma, with both spindle cell elements and squamous islands with focal keratinization $(4,5)$. We now describe the variable clinical and pathologic presentations of 31 patients with SCSC arising in association with TCV. In the most common clinical scenario (type I), both TCV and SCSC components are present in the initial resection. In the second scenario (type II), SCSC arises as a recurrence in a patient with a known history of TCV. In the third clinical presentation, SCSC presents as a mimic of primary laryngeal squamous cell carcinoma in a patient who may or may not have a known history of TCV. These data indicate that a diagnosis of $\mathrm{TCV}$ is clinically relevant for pathologic evaluation of both the initial resection and

\footnotetext{
${ }^{1}$ Department of Pathology and Laboratory Medicine, University of Pennsylvania School of Medicine, Philadelphia, Pennsylvania.

${ }^{2}$ Department of Pathology, Thomas Jefferson University, Philadelphia, Pennsylvania.
} 
recurrences, which may contain anaplastic SCSC. This is an important consideration in the differential diagnosis because SCSC may occur as a primary tumor in the oropharyngeal region. Further, patients with a history of TCV PTC who present with a presumed primary laryngeal squamous cell carcinoma should be evaluated for the possibility of a recurrence of TCV with transformation to SCSC, particularly if the patient does not have other risk factors for head and neck carcinogenesis.

\section{Materials and Methods}

This study was approved by the University of Pennsylvania Institutional Review Board. The cases were selected from the Surgical Pathology files from the Hospital of the University of Pennsylvania (20 cases) and the consultation files of one of the authors (V.A.L.; 11 cases). Clinical information was obtained through chart review, and the available pathologic history was reviewed for each case. Hematoxylin and eosinstained sections were reviewed. In some of the type II and type III cases, the histology of the primary tumor and a few of the recurrences (type II cases only; see below) were not available for review since many of these specimens were at other institutions and from several years ago. In some of the cases, immunostains were available; these stains included cytokeratins, thyroid transcription factor 1 (TTF-1), and thyroglobulin.

\section{Results}

In all cases, the tall cell papillary component displayed long, well-formed papillae with fibrovascular cores lined by tall columnar cells with pink cytoplasm, in which the cell height was two to three times the cell width (Fig. 1A). In addition, the cells had typical nuclei of papillary carcinoma, with enlarged oval and overlapping nuclei showing central clearing and ground glass chromatin. Nuclear grooves and multiple intranuclear inclusions, which give rise to the characteristic "soap bubble" intranuclear inclusions characteristic of TCV on cytologic preparations (6), were also observed. The SCSC anaplastic component showed spindle cells as well as solid nests and infiltrating islands of moderately differentiated squamous cell carcinoma, some of which had bland cytology (Fig. 1B). Other areas showed anaplastic spindle cells with a high nuclear:cytoplasmic ratio, pleomorphic, hyperchromatic nuclei with irregular contours, and prominent nucleoli.

The clinical and pathologic findings are summarized in Tables 1-3. The average age at presentation was 67 (range 32-92) with a female-to-male ratio of 2:1. Patients found to have SCSC associated with TCV presented in three clinical and pathologic scenarios. Clinically, the majority of patients simply presented with a thyroid mass (18 patients); histologically, these cases were characterized by a mixture of TCV PTC and SCSC present concurrently in the thyroidectomy specimen (type I). Both tumor morphologies were intimately admixed, with areas of transition from TCV PTC to the spindled and squamous appearance of the anaplastic component. The transition zone was notable for areas of hemorrhage, and the squamous cells, some of which appear bland, merge with more hyperchromatic, pleomorphic nuclei of the anaplastic squamous and spindled cells. Extrathyroidal extension and lymphovascular invasion were seen in 14 and 8 (14/18 and 8/18), respectively, of the type I cases.
The tumors in the second group of cases (5 patients; type II) initially demonstrated TCV PTC in the thyroid resection, but the anaplastic SCSC developed only in recurrences or metastases, months to years later. In both cases 7 and 9 (Table 2), the transformation to SCSC occurred longer than 15 years after the diagnosis of TCV. The site of recurrence with SCSC transformation was most often in neck lymph nodes. Excision of these enlarged lymph nodes demonstrated metastatic TCV PTC with solid squamoid nests and pleomorphic spindled areas, consistent with SCSC transformation. In addition to anaplastic transformation in neck lymph nodes, one patient also had spindle-cell anaplastic carcinoma in an endobronchial mass (Case 28, Table 2). Metastatic TCV PTC in multiple pulmonary nodules was noted in another case; however, SCSC transformation was not seen in the lung wedge resection (Case 9, Table 2).

In the third clinical scenario (type III), eight patients presented with a laryngeal mass (Fig. 2), and in four cases had a biopsy showing invasive squamous cell carcinoma before laryngectomy. The smoking history was only available for two of these patients (Cases 15 and 29). Whereas one of the patients (Case 15) had a 20 pack-year smoking history at the time of presentation with the laryngeal mass, the other patient (Case 29) was a life-long nonsmoker and had no obvious risk factors for head and neck carcinogenesis. Five of the eight patients had a prior history of PTC, and three of these five patients were known to have had TCV. In all cases, however, evaluation of the laryngectomy specimen revealed anaplastic SCSC arising in association with TCV PTC. The lack of an associated in situ squamous carcinoma or dysplasia in the laryngeal mucosa argued against a primary laryngeal squamous cell carcinoma in these cases. In addition, the intimate association of the TCV and SCSC components, with transitions between PTC to SCSC, strongly suggests that both morphologies are components of one tumor, rather than from the collision of two tumors. Although not routinely available before 2000, TTF-1 immunoreactivity is also helpful for distinguishing between these possibilities. The expression of TTF-1 by the squamous anaplastic component is strong evidence that the squamous cells are derived from follicular cells (Fig. 3) and are less likely to represent a concurrent or metastatic squamous carcinoma colliding with PTC.

\section{Discussion}

TCV of PTC, first described in 1976, is distinguished from other types of PTC by its tall columnar cell shape, with a height:width ratio of 2-3:1, and abundant eosinophilic or oxyphilic cytoplasm $(1,2)$. The accurate classification of TCV is clinically relevant, since these tumors tend to behave more aggressively $(7,8)$, and TCV may transform to SCSC anaplastic carcinoma $(4,5)$. We now describe the variable clinical presentations of 31 patients with anaplastic SCSC arising from TCV PTC and the clinical and pathologic considerations for diagnosis of these unusual tumors. Based on histologic examination, each case was classified into one of three clinical presentations: type I, in which TCV and SCSC are both identified in the initial resection (18 patients); type II, in which SCSC arises as a recurrence/metastasis in patients with a known history of TCV (5 patients); and type III, in which SCSC presents as a primary laryngeal squamous cell carcinoma in a patient with or without a known history of TCV (8 patients). 


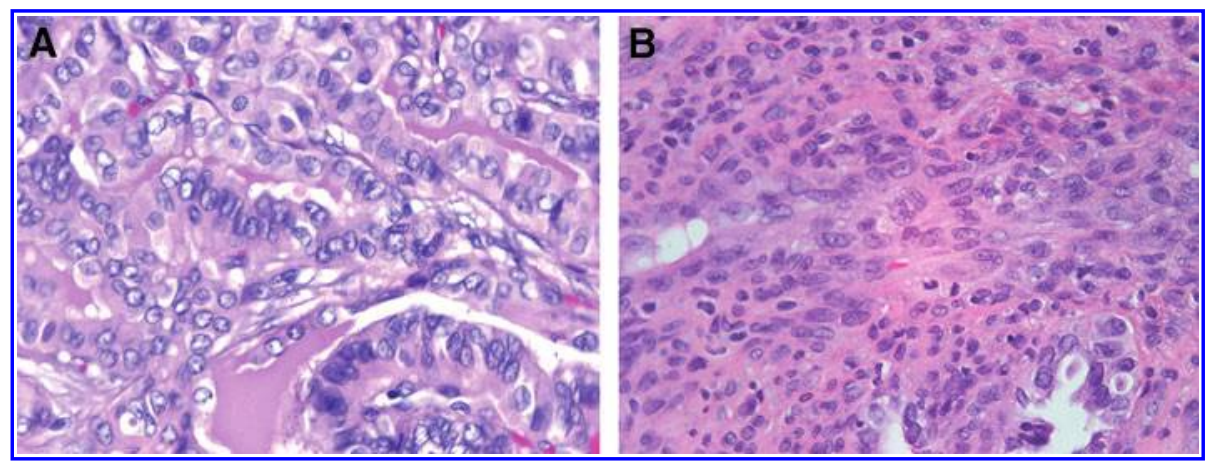

FIG. 1. (A) Tall cell variant papillary thyroid carcinoma is characterized by well-formed papillae lined by tall columnar cells with pink cytoplasm and cell height two to three times the cell width. The cells also have typical nuclei of papillary carcinoma, with enlarged oval and overlapping nuclei showing central clearing, ground glass chromatin, and occasional nuclear grooves. (B) The transition zone between tall cell variant and the anaplastic component shows solid areas of squamous cells, some of which have bland cytology.

Most commonly, patients with SCSC arising from TCV present with both tumor morphologies in close apposition in the initial thyroid resection specimen (type I) and present with a clinical thyroid mass. In contrast, the type II cases had a history of TCV or prior thyroidectomy with TCV PTC; months to years later, however, these patients presented with recurrences, most often in cervical lymph nodes, that showed metastatic TCV with dedifferentiation and transformation to SCSC. In both the type I and II cases, the histology demonstrates areas of transition from the well differentiated tall cell morphology, to more poorly differentiated, and spindle and squamous anaplastic morphology, and this intimate relationship leaves little diagnostic question that the SCSC arose in association with TCV PTC.

The type III cases presented very differently, with a laryngeal mass; four of eight patients had a biopsy diagnosed as invasive squamous cell carcinoma or poorly differentiated malignant neoplasm. These patients were presumed clinically to have a primary laryngeal squamous cell carcinoma. Only five of these patients had a history of PTC (three of who were found to have TCV PTC). In these cases, the clinical presentation of a laryngeal mass and the length of time (ranging

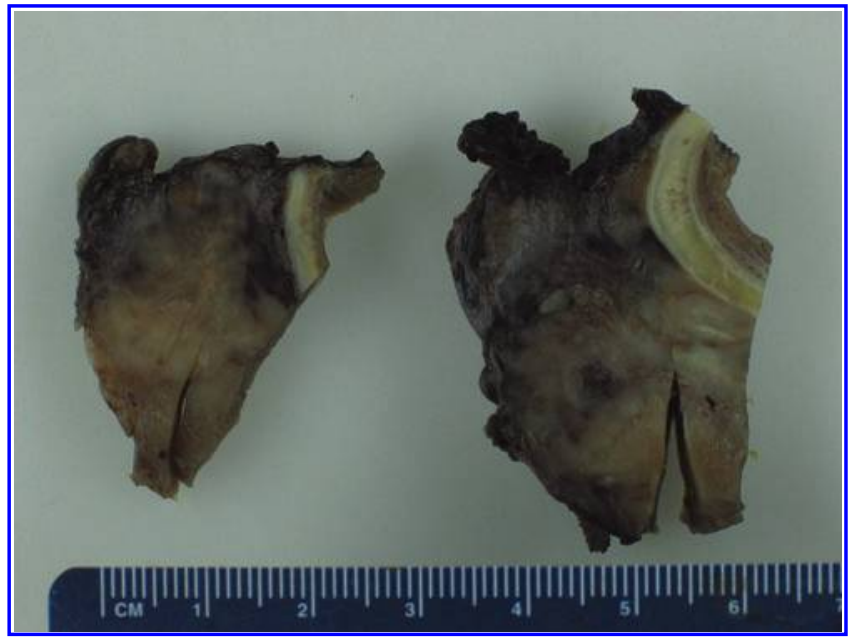

FIG. 2. Gross photograph of laryngectomy specimen from Case 17, bisected to reveal a poorly circumscribed solid tumor mass involving the infraglottic space. from 2 to 15 years) from the apparently cured PTC led to the assumption that the new mass was a primary squamous carcinoma of the larynx. Hence, all type III patients underwent layrngectomy. However, histologic evaluation of the laryngectomy specimens showed anaplastic SCSC admixed with areas of TCV of papillary carcinoma.

The differential diagnosis includes a collision of a primary laryngeal SCSC with a recurrent PTC, or SCSC arising in association with TCV PTC. In all cases, the lack of an associated in situ squamous carcinoma or dysplasia argues against a primary laryngeal squamous cell carcinoma. In addition, the intimate association of the TCV and SCSC components, with transitions between TCV PTC to SCSC, strongly suggests that both components arose from one tumor, rather than from the collision of two tumors. TTF-1 positivity in the SCSC component in more recently diagnosed cases helps determine the primary origin of these tumors from the pre-existing TCV. (TTF-1 immunohistochemistry was not performed in all cases, as tissue blocks were not always available [consult cases], and in cases before 2000, TTF-1 immunostaining was not routinely available.)

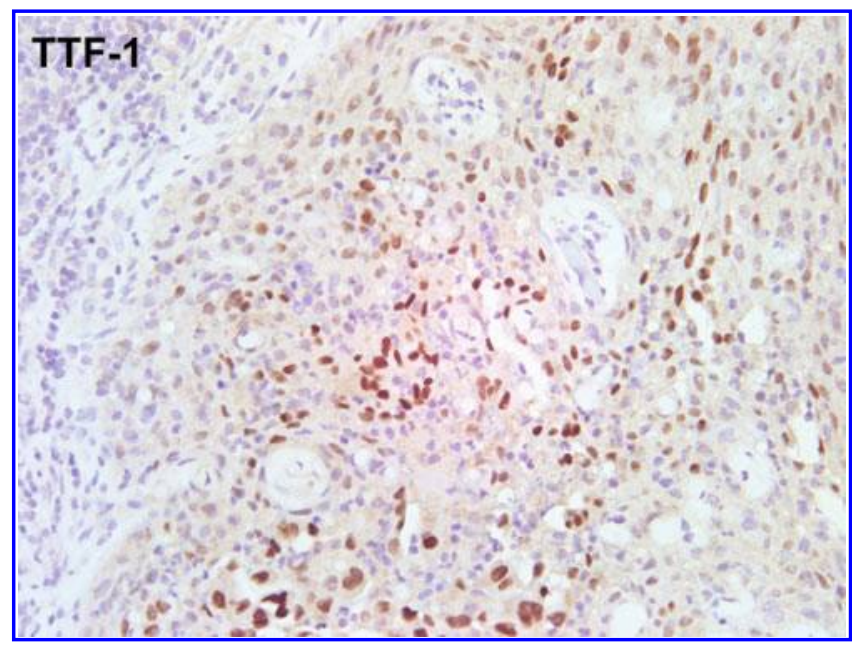

FIG. 3. TTF-1 immunostaining of the squamous anaplastic component strongly supports the thyroid follicular origin of these cells, rather than a concurrent primary squamous carcinoma of the larynx (collision tumor). TTF-1, thyroid transcription factor 1 . 
Table 1. Summary of Clinical and Pathologic Findings in Anaplastic Spindle Cell Squamous Carcinoma Arising in Tall Cell Variant (Type 1)

Case Age/gender

Summary of clinical and pathologic findings

$159 / \mathrm{M}$

History of astrocytoma status postresection 10 years before presentation and hemi-thyroidectomy 5 years before presentation (by history said to be benign lesion); presents with 1 month of stridor, dysphagia, hoarseness, and large left thyroid mass with compression of trachea.

Pathology: Anaplastic SCSC arising from TCV PTC, with invasion into perithyroidal soft tissue, parathyroid, and skeletal muscle; +LVI, +PNI.

59/F Former smoker with emphysema presents with worsening dyspnea and left neck mass; also found to have left thyroid lesion and numerous bilateral pulmonary nodules. Patient died of disease 12/2007.

Pathology: TCV PTC with extrathyroidal extension, extensive necrosis, and two de-differentiated foci (anaplastic carcinoma); +LVI.

51/F Left thyroid mass discovered incidentally during evaluation for hypercalcemia and parathyroid adenoma; status post-thyroidectomy and radioactive iodine.

Pathology: Anaplastic SCSC arising from TCV PTC, with extrathyroidal extension, + PNI.

78/M Primary MD noted a thyroid nodule during evaluation/treatment for a parathyroid adenoma.

Pathology: Anaplastic SCSC (25\% of tumor) arising from TCV PTC, with extensive necrosis; +LVI.

6 59/F Presents with thyroid mass.

Pathology: TCV PTC and areas of anaplastic transformation, extensively involving skeletal muscle and adipose tissue; +LVI.

54/M Presents with a right thyroid mass compressing the trachea.

Pathology: PTC, TCV and follicular variant, and anaplastic transformation (spindle and squamoid features), with extensive extrathyroidal spread into the trachea, lymphatics, skeletal muscle, + PNI.

63/F Presents with pain and an enlarging thyroid mass over 2-3 months.

Pathology: TCV PTC with anaplastic transformation.

56/F Presents with a thyroid mass and tracheal compression.

Pathology: Anaplastic SCSC arising from TCV PTC, with extrathyroidal extension.

18 54/M Presents with thyroid mass.

Pathology: Anaplastic SCSC arising from TCV PTC; +LVI.

19 68/F Presents with thyroid nodule.

Pathology: TCV PTC with anaplastic transformation to SCSC $(<5 \%)$, with extensive extrathyroidal extension into soft tissue and skeletal muscle, +LVI.

20 57/M Presents with thyroid nodule.

Pathology: Anaplastic SCSC arising from TCV PTC.

21 76/F Presents with thyroid mass.

Pathology: Anaplastic SCSC arising from TCV PTC, with extrathyroidal extension and invasion of skeletal muscle.

22 74/F History of Graves' disease status postradioactive iodine; presents with neck mass.

Pathology: TCV PTC with squamous differentiation, involving extrathyroidal soft tissue and skeletal muscle, +LVI.

82/M History of Graves' disease status postradioactive iodine (2004); presents with hoarseness and enlarging right neck mass over 2 months.

Pathology: Anaplastic SCSC arising in association with TCV PTC, with extrathyroidal spread.

24 92/F Presents with dyspnea and hoarseness over 2 years; U/S of the neck revealed a $4 \times 3.1 \mathrm{~cm}$ right thyroid lobe which displaced the trachea and was associated with paralysis of the right true vocal cord.

Pathology: TCV PTC extensively infiltrative in thyroid, extrathyroidal soft tissues, + LVI and with transformation to anaplastic SCSC $(<5 \%)$.

59/F History of left breast cancer status postradiation in 1999; presents 8 years later with a thyroid mass.

Pathology: SCSC arising in association with TCV PTC, with extensive extrathyroidal extension and invasion of skeletal muscle.

26 85/F Presents with a rapidly growing thyroid mass.

Pathology: Anaplastic SCSC arising in association with TCV PTC and Warthins like variant, with extensive extrathyoidal extension.

31 76/F Presents with shortness of breath; imaging shows a rapidly growing thyroid/laryngeal mass.

Pathology: Anaplastic SCSC arising in association with TCV PTC, with extensive necrosis, extrathyoidal extension, extension to laryngotracheal area, and with metastases to regional lymph nodes.

SCSC, spindle cell squamous carcinoma; TCV, tall cell variant; PTC, papillary thyroid carcinoma; LVI, lymphovascular invasion; PNI, perineural invasion. 
Table 2. Summary of Clinical and Pathologic Findings in Anaplastic Spindle Cell Squamous Carcinoma Arising in Patients with a History of Papillary Thyroid Carcinoma and Tall Cell Variant (Type 2)

\begin{tabular}{|c|c|c|}
\hline Case & Age/gender & Summary of clinical and pathologic findings \\
\hline 3 & $32 / \mathrm{F}$ & $\begin{array}{l}\text { History of PTC status post-thyroidectomy, subsequent bilateral neck and paratracheal dissections. } \\
\text { Pathology: PTC with focal tall cell features }(<10 \%) \text { in } 1991 \text {; multiple recurrences of TCV PTC } \\
\text { in lymph nodes, with development of squamoid features and solid nests, consistent with } \\
\text { anaplastic SCSC transformation in } 2007 \text {. }\end{array}$ \\
\hline 9 & $68 / \mathrm{F}$ & $\begin{array}{l}\text { Thyroidectomy in } 1979 \text { and subsequently had multiple right neck masses and bilateral } \\
\text { pulmonary nodules. } \\
\text { Pathology: Right neck lymph nodes with metastatic TCV PTC in 1993; right neck lymph nodes in } 1996 \\
\text { and 2001 show TCV PTC with focal anaplastic transformation and right lower lobe lung wedge in } \\
1998 \text { shows TCV PTC. }\end{array}$ \\
\hline 10 & 78/M & $\begin{array}{l}\text { Initial biopsy of thyroid mass shows TCV PTC. Presents with neck mass surrounding the carotid artery } \\
\text { and large veins. } \\
\text { Pathology: TCV PTC, invading skeletal muscle and +PNI, with more poorly differentiated areas } \\
\text { of tumor showing spindled appearance. }\end{array}$ \\
\hline 28 & $74 / \mathrm{F}$ & $\begin{array}{l}\text { History of a thyroid carcinoma who presents with a neck mass in } 2001 \text { and a recurrent neck mass } \\
\text { in 2007. In 2010, she presents with an endobronchial mass. } \\
\text { Pathology: Neck mass (2001) shows recurrent PTC with tall cell features; recurrent neck mass (2007) } \\
\text { shows a lymph node with metastatic papillary carcinoma with focal necrosis and squamous } \\
\text { differentiation. The endobronchial mass shows SCSC anaplastic carcinoma. }\end{array}$ \\
\hline 30 & $72 / \mathrm{M}$ & $\begin{array}{l}\text { History of papillary thyroid carcinoma in } 1980 \text { and nodal recurrence a few years later. In 1985, } \\
\text { he developed an ulcerating tracheal/cervical mass. Patient died of disease shortly thereafter. } \\
\text { Pathology: Cervical mass (1985) shows recurrent TCV PTC and anaplastic SCSC. }\end{array}$ \\
\hline
\end{tabular}

Table 3. Summary of Clinical and Pathologic Findings in Anaplastic Spindle Cell Squamous Carcinoma Presenting As a Laryngeal Mass in Patients With or Without a Known History of Tall Cell Variant Papillary Thyroid Carcinoma (Type 3)

Case Age/gender Summary of clinical and pathologic findings

$7 \quad$ 75/F History of TCV PTC, presents with laryngeal mass.

Pathology: Recurrent TCV PTC with transition to multifocal poorly differentiated areas with squamoid or spindle cells, consistent with anaplastic SCSC transformation.

8 74/M Presents with laryngeal mass.

Pathology: Anaplastic SCSC arising from TCV PTC, invading soft tissue between the trachea and esophagus.

$1168 / \mathrm{F}$ Presents with a larynx mass.

Pathology: TCV PTC with squamous change and associated focal anaplastic carcinoma; there is no mucosal in situ component associated with this tumor, supporting the impression that these squamoid areas represent squamous differentiation of thyroid carcinoma, rather than a separate primary squamous cell carcinoma of the larynx.

12 69/F History of PTC at an outside hospital in 1994; two years later presents with a left subglottic mass.

Pathology: Total laryngectomy specimen-anaplastic thyroid carcinoma with foci of TCV PTC, extending into the muscle. The tumor is comprised of plump spindle-shaped cells with large pleomorphic nuclei. There is no evidence of evolution from the epithelium.

$1577 / \mathrm{M} 20$ pack-year smoker with history of TCV PTC in 1997; presents four years later with a supraglottic mass.

Pathology: Total laryngectomy specimen-TCV PTC with anaplastic transformation.

17 58/M Presents with thyroid and laryngeal mass.

Pathology: Total laryngectomy specimen-Anaplastic SCSC arising from TCV PTC, replacing the entire left lobe and isthmus of thyroid, with invasion into extrathyroidal soft tissue, skeletal muscle, laryngeal/thyroid cartilages, and subglottic laryngeal mucosa.

27 72/F Life-long non-smoker with a history of PTC 15 years before; presents with a larynx mass.

Pathology: Anaplastic SCSC arising in association with TCV PTC, recurrent by history.

$2950 / \mathrm{F}$ History of TCV PTC, $5.7 \mathrm{~cm}$, in 2007 with extrathyroidal extension and invasion into trachea and cricoid cartilage with lymph node metastases with recurrence in larynx in 2009.

Pathology (2009): Anaplastic SCSC infiltrating thyroid and cricoid cartilages with areas of TCV and lymph node metastases with TCV. 
TTF-1 reactivity in the SCSC component does support a thyroid origin but may also be seen in other primary squamous tumors, such as lung $(9,10)$. In addition, thyroglobulin is negative, except at the tumor edge due to diffusion artifact. Immunohistochemistry for other markers may not be helpful in distinguishing between a primary laryngeal squamous cell carcinoma and SCSC. The squamous components of SCSC are usually positive for low- and high-molecular-weight cytokeratins, and the spindle cells are weakly and variably positive for low-molecular-weight cytokeratins and cytokeratins $903 / 904$ (4). In the future, molecular techniques such as differential microRNA profiles between primary laryngeal squamous carcinoma and anaplastic SCSC may help distinguish between these tumors (11-13).

Bronner and LiVolsi suggested the association of these two unusual tumors may represent an interesting histopathologic link in the pathology of the thyroid (4). Although several authors subsequently have proposed a step-wise progression from well-differentiated papillary carcinoma to poorly differentiated carcinoma, or to anaplastic carcinoma, the pathogenesis of this dedifferentiation is poorly understood (14-16). The RAS mutations have been implicated in the progression of papillary carcinoma to poorly differentiated carcinoma (16). Recent studies have shown that $B R A F$ is mutated at codon 599 in $40 \%$ or more of PTCs (17-19). BRAF mutations may be enriched in TCV PTC; one study demonstrated that all six TCV PTC cases harbored the BRAF mutation (20). In addition, anaplastic carcinomas (two of three cases, and three of nine cases) with adjacent TCV PTC also tested positive for the $B R A F$ mutation $(20,21)$. However, BRAF mutations are seen at all stages of PTC, including papillary microcarcinomas, indicating that additional mutations must take place for dedifferentiation to occur. Acquisition of p53 mutations may be one such event in the progression of $B R A F$-mutated papillary carcinomas to anaplastic carcinoma (19).

In summary, patients found to have SCSC anaplastic carcinoma arising from TCV of PTC may present in various clinical scenarios. The clinical history of TCV is very important, especially when patients present with recurrences or metastases that have dedifferentiation of the primary tumor. If a patient with a history of TCV presents with a laryngeal mass, clinicians and pathologists should consider SCSC arising from TCV PTC in the differential diagnosis, particularly if the patient does not have a smoking history or other risk factors for head and neck carcinogenesis.

\section{Disclosure Statement}

The authors declare that no competing financial interests exist.

\section{References}

1. Hawk WA, Hazard JB 1976 The many appearances of papillary carcinoma of the thyroid. Cleveland Clin Q 43:207216.

2. Ghossein R, LiVolsi VA 2008 Papillary thyroid carcinoma tall cell variant. Thyroid 18:1179-1181.

3. Ostrowski ML, Merino MJ 1996 Tall cell variant of papillary thyroid carcinoma: a reassessment and immunohistochemical study with comparison to the usual type of papillary carcinoma of the thyroid. Am J Surg Pathol 20:964-974.
4. Bronner MP, LiVolsi VA 1991 Spindle cell squamous carcinoma of the thyroid: an unusual anaplastic tumor associated with tall cell papillary cancer. Mod Pathol 4:637-643.

5. Kleer CG, Giordano TJ, Merino MJ 2000 Squamous cell carcinoma of the thyroid: an aggressive tumor associated with tall cell variant of papillary thyroid carcinoma. Mod Pathol 13:742-746.

6. Solomon A, Gupta PK, LiVolsi VA, Baloch ZW 2002 Distinguishing tall cell variant of papillary thyroid carcinoma from usual variant of papillary thyroid carcinoma in cytologic specimens. Diagn Cytopathol. 27:143-148.

7. Johnson TL, Lloyd RV, Thompson NW, Beierwaltes WH, Sisson JC 1988 Prognostic implications of the tall cell variant of papillary thyroid carcinoma. Am J Surg Pathol 12:22-27.

8. Prendiville S, Burman KD, Ringel MD, Shmookler BM, Deeb ZE, Wolfe K, Azumi N, Wartofsky L, Sessions RB 2000 Tall cell variant: an aggressive form of papillary thyroid carcinoma. Otolaryngol Head Neck Surg 122:352-357.

9. Bejarano PA, Baughman RP, Biddinger PW, Miller MA, Fenoglio-Preiser C, al-Kafaji B, Di Lauro R, Whitsett JA 1996 Surfactant proteins and thyroid transcription factor- 1 in pulmonary and breast carcinomas. Mod Pathol 9:445-452.

10. Harlamert HA, Mira J, Bejarano PA, Baughman RP, Miller MA, Whitsett JA, Yassin R 1998 Thyroid transcription factor1 and cytokeratins 7 and 20 in pulmonary and breast carcinoma. Acta Cytol 42:1382-1388.

11. Visone R, Pallante P, Vecchione A, Cirombella R, Ferracin M, Ferraro A, Volinia S, Coluzzi S, Leone V, Borbone E, Liu CG, Petrocca F, Troncone G, Calin GA, Scarpa A, Colato C, Tallini G, Santoro M, Croce CM, Fusco A 2007 Specific microRNAs are downregulated in human thyroid anaplastic carcinomas. Oncogene 26:7590-7595.

12. Long XB, Sun GB, Hu S, Liang GT, Wang N, Zhang XH, Cao PP, Zhen HT, Cui YH, Liu Z 2009 Let-7a microRNA functions as a potential tumor suppressor in human laryngeal cancer. Oncol Rep 22:1189-1195.

13. Kimura S, Naganuma S, Susuki D, Hirono Y, Yamaguchi A, Fujieda S, Sano K, Itoh H 2010 Expression of microRNAs in squamous cell carcinoma of human head and neck and the esophagus: miR-205 and miR-21 are specific markers for HNSCC and ESCC. Oncol Rep 23:1625-1633.

14. Asakawa H, Kobayashi T 2002 Multistep carcinogenesis in anaplastic thyroid carcinoma: a case report. Pathology 34: 94-97.

15. Wiseman SM, Loree TR, Hicks WL Jr., Rigual NR, Winston JS, Tan D, Anderson GR, Stoler DL 2003 Anaplastic thyroid cancer evolved from papillary carcinoma: demonstration of anaplastic transformation by means of the inter-simple sequence repeat polymerase chain reaction. Arch Otolaryngol Head Neck Surg 129:96-100.

16. Volante M, Rapa I, Gandhi M, Bussolati G, Giachino D, Papotti M, Nikiforov YE 2009 RAS mutations are the predominant molecular alteration in poorly differentiated thyroid carcinomas and bear prognostic impact. $\underline{\text { Clin }}$ Endocrinol Metab 94:4735-4741.

17. Kimura ET, Nikiforova MN, Zhu Z, Knauf JA, Nikiforova YE, Fagin JA 2003 High prevalence of BRAF mutations in thyroid cancer: genetic evidence for constitutive activation of RET/PTC-RAS-BRAF signaling pathway in papillary thyroid carcinoma. Cancer Res 63:1454-1457.

18. Cohen Y, Xing M, Mambo E, Guo Z, Wu G, Trink B, Beller U, Westra WH, Ladenson PW, Sidransky D 2003 BRAF mutation in papillary thyroid carcinoma. J Natl Cancer Inst 95:625-627. 
19. Quiros RM, Ding HG, Gattuso P, Prinz RA, Xu X 2005 Evidence that one subset of anaplastic thyroid carcinomas are derived from papillary carcinomas due to BRAF and p53 mutations. Cancer 103:2261-2268.

20. Nikiforova MN, Kimura ET, Gandhi M, Biddinger PW, Knauf JA, Basolo F, Zhu Z, Giannini R, Salvatore G, Fusco A, Santoro M, Fagin JA, Nikiforov YE 2003 BRAF mutations in thyroid tumors are restricted to papillary carcinomas and anaplastic or poorly differentiated carcinomas arising from papillary carcinomas. J Clin Endocrinol Metab 88:5399-5404.

21. Takano T, Ito Y, Hirokawa M, Yoshida H, Miyauchi A 2007 BRAF V600E mutation in anaplastic thyroid carcinomas and their accompanying differentiated carcinomas. Br J Cancer 96:1549-1553.

Address correspondence to: Pallavi P. Gopal, M.D., Ph.D. Department of Pathology and Laboratory Medicine University of Pennsylvania School of Medicine 3400 Spruce St. Philadelphia, PA 19104

E-mail: pallavi.gopal@uphs.upenn.edu 
